\title{
Correlation Analysis of Thyroid Function and Autoantibody in Healthy Population Living in the Area of Water-Source-Originated High Level lodine
}

\author{
Hou Zhenjiang ${ }^{1, *}$, Mu Zhaoxin ${ }^{1}$, Wang Fengling ${ }^{1}$, Li Hongyan ${ }^{1}$, Zhang Jingyu ${ }^{2}$, Fan Hong ${ }^{2}$, \\ Wang Huaxin ${ }^{3}$ \\ ${ }^{1}$ Institute of Thyroid Diseases, Cangzhou Medical College, Cangzhou, China \\ ${ }^{2}$ Laboratory Diagnostics Division, Hebei Cangzhou Hospital of Integrated Traditional Chinese and Western Medicine, Cangzhou, China \\ ${ }^{3}$ Hebei Huanghua Development Zone Bo'ai Hospital, Huanghua, China
}

Email address:

houzhenjiang@sina.com (Hou Zhenjiang)

${ }^{*}$ Corresponding author

\section{To cite this article:}

Hou Zhenjiang, Mu Zhaoxin, Wang Fengling, Li Hongyan, Zhang Jingyu, Fan Hong, Wang Huaxin. Correlation Analysis of Thyroid Function and Autoantibody in Healthy Population Living in the Area of Water-Source-Originated High Level Iodine. Science Journal of Public Health . Vol. 5, No. 3, 2017, pp. 198-204. doi: 10.11648/j.sjph.20170503.17

Received: January 20, 2017; Accepted: March 1, 2017; Published: March 20, 2017

\begin{abstract}
In order to know about the correlation between thyroid function and autoantibody of urban residents in Cangzhou, the author chose 3218 health examinations as the respondents from Cangzhou to do a research. First of all, fasting venous blood was taken from elbow in the morning. Secondly, serum was separated by centrifugation. Moreover, the positive rates of TSH, FT3, FT4, TPOAb and TgAb in serum were tested by use of Germany Roche chemiluminescence immune detection system. It finally turns out that thyroid dysfunction and positive rate of autoantibody are $7.71 \%$ and $16.19 \%$, respectively, which have been increasing sharply along with the growth of the age, and the positive rate of female is higher than that of the male $(\mathrm{P}<0.001)$. Besides, the prevalence rates of hyperthyroidism, subclinical hyperthyroidism, hypothyroidism, and subclinical hypothyroidism are $0.81 \%, 0.68 \%, 1.83 \%$ and $4.38 \%$, respectively. The prevalence rate of subclinical thyroid disease $(5.07 \%)$ is significantly higher than that of clinical thyroid disease (2.64\%), and the prevalence rate of clinical hypothyroidism is the highest, accounting for $56.9 \%$ of thyroid dysfunction. Positive rates of TPOAb and TgAb are $14.57 \%$ and $12.74 \%$, respectively, while the simultaneous positive detection rate of TPOAb and TgAb is $11.12 \%$. Through the analysis of test data, the author comes to the conclusion that thyroid dysfunction and positive rate of autoantibody have been increasing year by year, which has a great relationship with gender and age. As a result, it is important to put the determination of thyroid function and autoantibody into the routine testing and it is necessary for normal thyroid function and positive autoantibody to conduct a dynamic monitoring, follow-up or intervention in time, which is beneficial to early diagnosis, differential diagnosis and prophylaxis and treatment.
\end{abstract}

Keywords: Thyroid Hormone, Thyroid Dysfunction, Thyroid Autoantibody, Health Examination

\section{Introduction}

Thyroid disease is a common and frequently-occurring disease in endocrine system. With the continuous improvement of detection method and the application and popularization of automatic analyzer, using sensitive and rapid electro-chemiluminescence immunoassay (ECLIA) determination to determine thyroid hormone has become the comparatively ideal choice and development trend [1].
Thyroid diseases, including hyperthyroidism, subclinical hyperthyroidism, hypothyroidism and subclinical hypothyroidism, with or without significant clinical manifestations, and laboratory tests are often accompanied by thyroid dysfunction. The diagnosis shall refer to the medical history, clinical manifestations and laboratory tests, especially the serum thyroid hormone test results [2].

The level of thyroid stimulating hormone (TSH), serum free triiodothyronine (FT3), serum free thyroxine (FT4), thyroid peroxidase antibody (TPOAb) and thyroglobulin antibody 
(TgAb) measured by ECLIA is very important for the diagnosis and differential diagnosis of thyroid diseases, especially the diagnosis of auto-immune thyroid diseases (AITD) [3] [4] [5]. The sensitivity and specificity of other joint detection of TSH, $\mathrm{TPOAb}$ and TgAb for the AITD and non-AITD differential diagnosis were higher than the single index. Therefore, the determination of serum thyroid hormone and autoantibody levels contribute to the diagnosis, differential diagnosis, efficacy observation and prognosis monitoring of AITD. Cangzhou City belongs to the water-source-originated high level iodine area [6], Guan Haixia and Li Yushu et al [7] [8] have conducted epidemiological investigation of thyroid disease and autoantibody in Huanghua, Cangzhou and other places. The paper focuses on the thyroid hormone and autoantibody detection in urban resident healthy population in Cangzhou, to provide objective basis for the prevention and treatment of thyroid and related diseases.

\section{Materials and Methods}

\subsection{General Information}

Collected the information of 3218 urban residents in Cangzhou, who performed physical examination, thyroid palpation, ultrasound, and thyroid function and autoantibody examination in physical examination center from January 2013 to December 2015, including 1686 women, 1532 men, with the average age of $(46.29 \pm 10.96)$ years old. The patients were divided into 6 groups according to their ages: 348 cases in 20-29 years, 485 cases in 30-39 years, 634 cases in 40-49 years, 781 cases in 50-59 years, 672 cases in 60-69 years and 298 cases larger than 70 years old. Inclusion criteria: (1) No family history, past history of thyroid disease or other autoimmune diseases; (2) No history of pituitary or hypothalamic disease; (3) No goiter or obvious nodules; (4) No thyroid ultrasound abnormalities; (5) Without taking drugs affecting thyroid hormone, such as phenytoin sodium; (6) No family history of hyperlipidemia, recently did not take hypolipidemic drugs; (7) No chronic diseases (diabetes, liver dysfunction, renal dysfunction, cardiac insufficiency, chronic infectious disease, etc.), malignant neoplasms; (8) pregnancy excluded.

\subsection{Laboratory Examination}

Healthy subjects were fasting for more than $12 \mathrm{~h}$, and took venous blood at 7:30 - 9:30 in the morning, separating serum timely. Determined the level of serum TSH, FT3, FT4, $\mathrm{TPOAb}$ and TGAb with Immuno-chemiluminescence Detector and supporting reagents produced by the German Roche Co., Ltd.. Diagnosis of thyroid disease is mainly based on test results of serum TSH, FT3 and FT4, the diagnostic criteria are as follows [9]: (1) hyperthyroidism: with hypothyroidism symptoms or signs, $\mathrm{TSH}<0.27 \mathrm{mIU} / \mathrm{L}$, FT3 $>7.1 \mathrm{pmol}$ and/or FT4 $>22 \mathrm{pmol} / \mathrm{L}$; (2) subclinical hyperthyroidism: without hyperthyroidism symptoms or signs, TSH $<0.27 \mathrm{mIU} / \mathrm{L}$, normal FT3 and FT4; (3) hypothyroidism: with hypothyroidism symptoms or signs, TSH $>4.2 \mathrm{mIU} / \mathrm{L}$, FT3 $<2.8 \mathrm{pmol} / \mathrm{L}$ and/or FT4 $<12 \mathrm{pmol} / \mathrm{L}$; (4) subclinical hypothyroidism: without hyperthyroidism symptoms or signs, TSH $>4.20 \mathrm{mIU} / \mathrm{L}$, normal FT3 and FT4; TPOAb and TGAb were positive of $>34 \mathrm{IU} / \mathrm{mL}$ and $>115 \mathrm{IU} / \mathrm{mL}$, respectively.

\subsection{Statistical Analysis}

Adopted SPSS 17.0 software to implement statistical analysis. Used $\chi^{2}$ test for comparison between groups of thyroid dysfunction and TPOAb, TGAb positive rate, as well as positive rates of autoantibody in a variety of thyroid diseases, with $\mathrm{P}<0.05$ for the statistically significant. The risk of thyroid dysfunction (OR) was calculated using the unconditional Logistic Regression in patients with positive thyroid autoantibody.

\section{Results}

\subsection{Comparison of Thyroid Dysfunction and Autoantibody Positive Rate of Healthy Subjects in Different Years}

The total prevalence of thyroid dysfunction in healthy subjects of this investigation was 7.71\% (248/3218). The prevalence of hyperthyroidism was $1.49 \%$ (hyperthyroidism and subclinical hyperthyroidism were $0.81 \%, 0.68 \%$, respectively), and the prevalence of hypothyroidism was $6.22 \%$ (hypothyroidism and subclinical hypothyroidism were $1.83 \%$ and $4.38 \%$, respectively). The ratio of hyperthyroidism and hypothyroidism was 1:4.2. The prevalence of clinical thyroid dysfunction $(2.64 \%, 85 / 3218)$ was significantly lower than the prevalence of subclinical thyroid dysfunction $(5.07 \%, 163 / 3218, \mathrm{P}<0.001)$. See Table 1 .

Table 1. Comparison of thyroid dysfunction and autoantibody positive rate of healthy subjects in different years [n (\%)].

\begin{tabular}{|c|c|c|c|c|c|c|c|c|}
\hline Year & $\mathbf{N}$ & $(\mathbf{M} / \mathbf{F})$ & $\begin{array}{l}\text { Average age } \\
\text { (Years) }\end{array}$ & Hyperthyroidism & $\begin{array}{l}\text { Subclinical } \\
\text { hyperthyroidism }\end{array}$ & Hypothyroidism & $\begin{array}{l}\text { Subclinical } \\
\text { hypothyroidism }\end{array}$ & Total \\
\hline 2013 & 879 & $(426 / 453)$ & $45.16 \pm 11.52$ & $3(0.34)$ & $2(0.23)$ & $6(0.68)$ & $13(1.48)$ & $24(2.73)$ \\
\hline 2014 & 1086 & $(514 / 572)$ & $46.33 \pm 9.89$ & $7(0.64)$ & $5(0.46)$ & $17(1.57)$ & $42(3.87)$ & $71(6.54)$ \\
\hline 2015 & 1253 & $(592 / 661)$ & $46.27 \pm 10.73$ & $16(1.28)$ & $15(1.20)$ & $36(2.87)$ & $86(6.86)$ & $153(12.21)$ \\
\hline In total & 3218 & $(1532 / 1686)$ & & $26(0.81)$ & $22(0.68)$ & $59(1.83)$ & $141(4.38)$ & $248(7.71)$ \\
\hline$\chi^{2}$ & & 3.759 & 2.086 & 6.189 & 9.498 & 14.427 & 37.726 & 69.443 \\
\hline$P$ & & 0.153 & 0.239 & 0.045 & 0.009 & 0.001 & $<0.001$ & $<0.001$ \\
\hline
\end{tabular}

The total positive rate of thyroid autoantibody in healthy subjects was $16.19 \%(521 / 3218)$, and the positive rate of $\mathrm{TPOAb}$ and $\mathrm{TgAb}$ was $14.57 \%$ and $12.74 \%$, respectively, and the simultaneous positive detection rate of TPOAb and $\mathrm{TgAb}$ was $11.12 \%$. The thyroid dysfunction, positive rate of $\mathrm{TPOAb}$ and $\mathrm{TgAb}$ and simultaneous positive detection rate of 
$\mathrm{TPOAb}$ and $\mathrm{TgAb}$ were significantly increased with the increase of year, and it was significantly different in 2013 compared with 2014 and $2015(P<0.001)$. The gender and average There was no statistically significant difference in groups of sex and average age $\left(\chi^{2}=3.759,208.6, P=0.153\right.$, 0.239 , respectively), and there was no significant difference in simultaneous positive detection rate of TPOAb and $\mathrm{TgAb}$ between 2014 and $2015(P=0.199)$. See Table 2 .

Table 2. Positive rate of TPOAb and TgAb and simultaneous positive detection rate of TPOAb and TgAb of healthy subjects in different years [n (\%)].

\begin{tabular}{|c|c|c|c|c|c|c|c|}
\hline Year & $\mathbf{N}$ & $(\mathbf{M} / \mathbf{F})$ & Average age (Years) & TPOAb & TgAb & \multicolumn{2}{|c|}{ TPOAb and TgAb } \\
\hline 2013 & 879 & $(426 / 453)$ & $45.16 \pm 11.52$ & $76(8.65)$ & $68(7.75)$ & $59(6.71)$ & 203(23.09) \\
\hline 2014 & 1086 & $(514 / 572)$ & $46.33 \pm 9.89$ & $154(14.18)$ & $137(12.62)$ & $126(11.60)$ & $417(38.40)$ \\
\hline 2015 & 1253 & $(592 / 661)$ & $46.27 \pm 10.73$ & $239(19.01)$ & $205(16.36)$ & $173(13.81)$ & $617(49.24)$ \\
\hline & $\chi^{2}$ & 3.759 & 2.086 & 45.236 & 34.588 & 26.677 & 149.256 \\
\hline & $P$ & 0.153 & 0.239 & $<0.001$ & $<0.001$ & $<0.001$ & $<0.001$ \\
\hline
\end{tabular}

\subsection{Comparison of Positive Rate of Thyroid Dysfunction in Different Sex and Age Groups of Healthy Subjects}

The ratio of male to female with thyroid dysfunction in healthy subjects was 1:5.4. The prevalence of male and female was the lowest in the age group of $20-29(2.4 \% \mathrm{VS}$
$6.63 \%$ ), while the highest prevalence of male appeared in the age group of $40-49(4.36 \%)$. For female, there were two peaks, in the age group of $40-49$ and larger than $70,15.48 \%$ and $16.99 \%$, respectively. See Table 3 .

Table 3. Comparison of detection rate of thyroid dysfunction in different sex and age groups [n (\%)].

\begin{tabular}{|c|c|c|c|c|c|c|c|c|c|}
\hline $\begin{array}{l}\text { Age group } \\
\text { (Year) }\end{array}$ & $\mathbf{N}(\mathbf{M} / \mathbf{F})$ & $\mathbf{n}$ & $\begin{array}{l}\text { Prevalence } \\
\text { rate }(\%)\end{array}$ & $\begin{array}{l}\text { Hyperthyroidism } \\
\text { (M/F) }\end{array}$ & $\begin{array}{l}\text { Subclinical } \\
\text { hyperthyroidism (M/F) }\end{array}$ & $\begin{array}{l}\text { Hypothyroidism } \\
\text { (M/F) }\end{array}$ & $\begin{array}{l}\text { Subclinical } \\
\text { hypothyroidism } \\
\text { (M/F) }\end{array}$ & $\chi^{2}$ & $\boldsymbol{P}$ \\
\hline $20 \sim 29$ & 348 & 16 & 4.60 & $1(0 / 1)$ & $1(1 / 0)$ & $3(1 / 2)$ & $11(2 / 9)$ & 3.551 & $=0.06$ \\
\hline $30 \sim 39$ & 485 & 31 & 6.39 & $3(1 / 2)$ & $2(1 / 1)$ & $10(2 / 8)$ & $16(3 / 13)$ & 8.330 & $<0.004$ \\
\hline $40 \sim 49$ & 634 & 65 & 10.25 & $8(3 / 5)$ & $9(2 / 7)$ & $17(3 / 14)$ & $31(5 / 26)$ & 21.200 & $<0.001$ \\
\hline $50 \sim 59$ & 781 & 64 & 8.19 & $7(2 / 5)$ & $5(1 / 4)$ & $13(3 / 10)$ & $39(8 / 31)$ & 18.005 & $<0.001$ \\
\hline $60 \sim 69$ & 672 & 41 & 6.10 & $4(1 / 3)$ & $3(1 / 2)$ & $11(2 / 9)$ & $23(2 / 21)$ & 19.381 & $<0.001$ \\
\hline$\geqslant 70$ & 298 & 31 & 10.40 & $3(1 / 2)$ & $2(1 / 1)$ & $5(1 / 4)$ & $21(2 / 19)$ & 14.655 & $<0.001$ \\
\hline In total & 3218 & 248 & 7.71 & $26(8 / 18)$ & $22(7 / 15)$ & $59(12 / 47)$ & $141(22 / 119)$ & 66.950 & $<0.001$ \\
\hline
\end{tabular}

Note: (1) Comparison of female positive rate of thyroid dysfunction in different age groups $\chi^{2}=15.457, P=0.009$; (2) Comparison of male positive rate of thyroid dysfunction in different age groups $\chi^{2}=3.979, \mathrm{P}=0.552$.

\subsection{Comparison of Positive Rate of Thyroid Autoantibody in Different Sex and Age Groups of Healthy Subjects}

The positive rate of TPOAb in male was significantly lower than that in female $(6.92 \%$ VS $21.53 \%, \chi 2=137.634$, $P<0.001$ ), increased along with the increase of age, while the highest positive rate in male was in the age group of 50-59 $(9.49 \%)$, and the positive rate peak in female was in the age group of larger than $70(28.10 \%)$. The positive rate of TGAb in male was significantly lower than that in female $(5.68 \%$ VS $19.16 \%, \quad \chi 2=131.169, P<0.001)$, while the highest positive rate in male was in the age group of 50-59 (7.32\%), and the highest positive rate in female was in the age group of larger than 70 reached $19.16 \%$. The simultaneous positive rate of $\mathrm{TPOAb}$ and $\mathrm{TgAb}$ was $11.12 \%$, and it was significantly lower in male than that in female $(5.03 \% \mathrm{VS}$ $\left.16.67 \%, \chi^{2}=110.002, P<0.001\right)$. while the highest positive rate in male was in the age group of 50-59 (5.15\%), and the positive rate peak in female was in the age group of larger than $70(20.26 \%)$. The differences of positive rate of TPOAb, $\mathrm{TGAb}$ and simultaneous positive rate of TPOAb and TgAb between age groups larger than 30 and younger than 30 in male and female were significant $(P<0.001)$. See Table 4 .

Table 4. Comparison of positive rate of thyroid autoantibody in different sex and age groups of healthy subjects [n (\%)].

\begin{tabular}{|c|c|c|c|c|c|c|c|c|c|}
\hline \multirow[b]{2}{*}{ Age Group } & \multirow[b]{2}{*}{$\mathbf{N}$} & \multicolumn{2}{|c|}{ TPOAb (positive) } & \multicolumn{2}{|c|}{ TgAb (positive) } & \multicolumn{2}{|c|}{ TPOAb and TgAb (positive) } & \multirow[b]{2}{*}{$\chi^{2}$} & \multirow[b]{2}{*}{$\boldsymbol{P}$} \\
\hline & & M & $\mathbf{F}$ & M & $\mathbf{F}$ & M & $\mathbf{F}$ & & \\
\hline $20 \sim 29$ & 348 & $6(3.59)$ & $19(10.50)$ & $7(4.19)$ & $24(13.26)$ & $5(1.80)$ & $15(8.29)$ & 23.012 & $<0.001$ \\
\hline 30 39 & 485 & $14(6.06)$ & $50(19.69)$ & $12(5.19)$ & $41(16.14)$ & $11(2.60)$ & $35(13.78)$ & 61.170 & $<0.001$ \\
\hline $40 \sim 49$ & 634 & $21(7.05)$ & $71(21.13)$ & $15(5.03)$ & $62(18.45)$ & $13(3.02)$ & $56(16.67)$ & 106.731 & $<0.001$ \\
\hline $50 \sim 59$ & 781 & $35(9.49)$ & $94(22.82)$ & $27(7.32)$ & $87(21.12)$ & $26(5.15)$ & $76(18.45)$ & 117.186 & $<0.001$ \\
\hline $60 \sim 69$ & 672 & $21(6.52)$ & $86(24.57)$ & $19(5.90)$ & $75(21.42)$ & $15(4.66)$ & $68(19.43)$ & 160.656 & $<0.001$ \\
\hline$\geqslant 70$ & 298 & $9(6.21)$ & $43(28.10)$ & $7(4.83)$ & $34(22.22)$ & $7(4.83)$ & $31(20.26)$ & 90.506 & $<0.001$ \\
\hline In total & 3218 & $106(6.92)$ & $363(21.53)$ & $87(5.68)$ & $323(19.16)$ & $77(5.03)$ & $281(16.67)$ & & \\
\hline
\end{tabular}

Note: (1) Comparison of total positive detection rate of thyroid autoantibody between different male and female $\chi^{2}=535.427, P<0.001$; (2) Comparison of female positive detection rate of thyroid autoantibody in different age groups $\chi^{2}=16.310, P=0.006$; (3) Comparison of female positive detection rate of thyroid autoantibody in different age groups $\chi^{2}=78.343, P<0.001$. 


\subsection{Comparison of Positive Rate of Thyroid Autoantibody in Different Age Groups of Healthy Subjects}

The positive rate of thyroid autoantibody in all age groups was significantly different $(P<0.05 \sim<0.001)$. See Table 5 .

Table 5. Comparison of positive rate of thyroid autoantibody in different age groups of healthy subjects.

\begin{tabular}{|c|c|c|c|c|c|c|c|}
\hline \multirow[b]{2}{*}{ Age Group } & \multirow[b]{2}{*}{$\mathbf{N}$} & \multicolumn{2}{|c|}{ TPOAb (positive) } & \multicolumn{2}{|c|}{ TgAb (positive) } & \multicolumn{2}{|c|}{ TPOAb and TgAb (positive + ) } \\
\hline & & $\chi^{2}$ & $\boldsymbol{P}$ & $\chi^{2}$ & $\boldsymbol{P}$ & $\chi^{2}$ & $P$ \\
\hline 20 29 & 348 & 6.210 & 0.013 & 8.802 & 0.003 & 4.493 & 0.034 \\
\hline $30 \sim 39$ & 485 & 19.605 & $<0.001$ & 14.894 & $<0.001$ & 11.459 & $<0.001$ \\
\hline $40 \sim 49$ & 634 & 25.253 & $<0.001$ & 26.652 & $<0.001$ & 24.652 & $<0.001$ \\
\hline $50 \sim 59$ & 781 & 25.086 & $<0.001$ & 27.735 & $<0.001$ & 22.282 & $<0.001$ \\
\hline $60 \sim 69$ & 672 & 40.813 & $<0.001$ & 33.610 & $<0.001$ & 33.797 & $<0.001$ \\
\hline$\geqslant 70$ & 298 & 24.782 & $<0.001$ & 18.984 & $<0.001$ & 15.939 & $<0.001$ \\
\hline
\end{tabular}

\subsection{Comparison of Positive Rate and OR of Autoantibody in Subjects with Thyroid Dysfunction}

hypothyroidism and subclinical hypothyroidism were significantly different $(P<0.001)$, with the highest of hypothyroidism, followed by hyperthyroidism, subclinical

The positive rate and OR of TPOAb, TgAb and hypothyroidism and subclinical hyperthyroidism. See table 6 . simultaneous positive rate of TPOAb and $\mathrm{TgAb}$ between groups of hyperthyroidism, subclinical hyperthyroidism,

Table 6. Comparison of positive rate and OR of autoantibody in subjects with thyroid dysfunction.

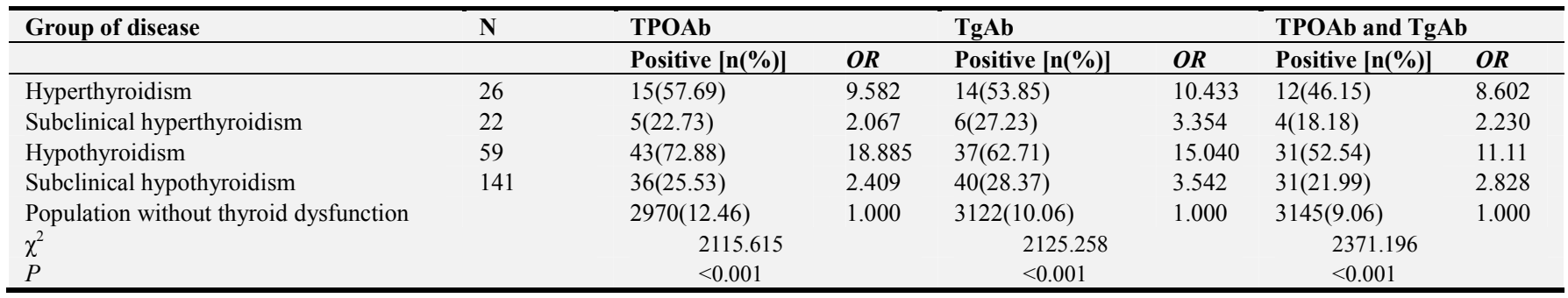

\section{Discussions}

The thyroid gland is the largest endocrine gland in the human body, and its secreted hormones play an important role in regulating human growth, development and metabolism. In recent years, with the changes in diet, increased mental stress, accelerated pace of life and the increase of environmental factors influencing on the thyroid function, the incidence of thyroid disease is on the rise. The study found that, the thyroid dysfunction, positive rate of TPOAb and TgAb and simultaneous positive detection rate of $\mathrm{TPOAb}$ and $\mathrm{TgAb}$ were significantly increased with the increase of year, and it was significantly different in 2013 compared with 2014 and $2015 \quad(P<0.001)$. The above-mentioned results are consistent with published literature [10] [11] [12], including that the incidence of thyroid disease and TPOAb, TGAb positive rate showed a significant rising trend, and the thyroid autoantibody baseline level of middle-aged and elderly people increased year by year, as well as there was a significant difference of $\mathrm{TgAb}$ and TPOAb between 3 years, with a trend of increase along with the year, which further confirmed that thyroid autoantibody was involved in the pathogenesis of AITD.

There were some differences in the epidemiological findings of thyroid disease among different authors. Guan Haixia et al [7] found that the prevalence of thyroid dysfunction in a certain village in Huanghua City was
$10.34 \%$, while the prevalence of hyperthyroidism, subclinical hyperthyroidism, hypothyroidism and subclinical hypothyroidism were $1.21 \%, 1.12 \%, 1.96 \%$ and $6.05 \%$, respectively. The ratio of prevalence of hyperthyroidism to hypothyroidism (including subclinical hypothyroidism) was 1:3.4. The prevalence of subclinical hypothyroidism was the highest, 1.41 times of other thyroid diseases. Yuan Shuai et al [13] reported that the prevalence of thyroid dysfunction in Shanghai residents was $3.98 \%$, while the prevalence of hyperthyroidism, subclinical hyperthyroidism, hypothyroidism and subclinical hypothyroidism were $0.47 \%$, $0.62 \%, 0.34 \%$ and $2.55 \%$, respectively. The ratio of prevalence of hyperthyroidism to hypothyroidism was 1:2.6. The prevalence of subclinical hypothyroidism was the highest, 1.78 times of other thyroid diseases. Wen Xiao heng et al [14] reported that the prevalence of thyroid dysfunction was $8.42 \%$, while the prevalence of hyperthyroidism, subclinical hyperthyroidism, hypothyroidism and subclinical hypothyroidism were $0.45 \%, 0.92 \%, 0.27 \%$ and $6.78 \%$, respectively. The ratio of prevalence of hyperthyroidism to hypothyroidism was 1:5.1. The prevalence of subclinical hypothyroidism was the highest, 4.13 times of other thyroid diseases. Results of this study showed that the prevalence of thyroid dysfunction was $7.71 \%$, while the prevalence of hyperthyroidism, subclinical hyperthyroidism, hypothyroidism and subclinical hypothyroidism were $0.81 \%$, $0.68 \%, 1.83 \%$ and $4.38 \%$, respectively. The ratio of prevalence of hyperthyroidism to hypothyroidism was 1:4.2. 
The prevalence of subclinical hypothyroidism was the highest, 1.32 times of other thyroid diseases, which is consistent with the highest prevalence of subclinical hypothyroidism [9]. There are some differences in the prevalence of thyroid dysfunction and the proportion of various thyroid diseases in different reports, which may be associated with the difference of the number of sample population, sex ratio, age distribution, iodine nutritional status, genetic background, geographical environment, detection method and diagnostic criteria and other factors, but most believed that hypothyroidism, especially the subclinical hypothyroidism had the highest prevalence, while female was higher than male.

The prevalence of thyroid dysfunction varies with sex and age. Results of this study showed that, the prevalence of thyroid dysfunction $(3.2 \%)$ was significantly lower in male than that in female $(11.80 \%, P<0.001)$, with a ratio of $1: 4.1$. The prevalence of hyperthyroidism, subclinical hyperthyroid -ism, hypothyroidism and subclinical hypothyroidism in female were $1.07 \%, 0.89 \%, 2.79 \%$ and $7.06 \%$, respectively, and those of male were $0.52 \%, 0.46 \%, 0.78 \%$ and $1.44 \%$ respectively. The prevalence of hyperthyroidism was higher than that of subclinical hyperthyroidism, with the highest prevalence of subclinical hypothyroidism, followed by subclinical hyperthyroidism, hypothyroidism, subclinical hypothyroidism, significantly lower in male than in female $(P<0.001)$. The lowest prevalence of thyroid dysfunction both in male and female was in the age group of $20-29(2.4 \%$ and $6.63 \%$, respectively), and the highest prevalence of male appeared in the age group of 40-49 (4.36\%), while for female, there were two peaks, in the age group of 40-49 and larger than $70,15.48 \%$ and $16.99 \%$, respectively, which is consistent with the report of $\mathrm{Wu}$ Xiaoqing et al [15], that is, the prevalence of male thyroid disease was significantly lower than that of women, increased with age, and differences in all age groups were statistically significant $(P<0.01)$.

Research material [16] [17] [18] indicated that, the prevalence of hypothyroidism was $6 \%-17 \%$, while female was higher than the male. With the increase of TSH in serum, the prevalence of hypothyroidism was $7.3 \%$ to $78 \%$ within 10 years. With the high sensitivity TSH detection technology, the prevalence of hyperthyroidism was as high as $20 \%$. The prevalence of hypothyroidism among the elderly was higher than that of the young, and the prevalence of hypothyroidism in the elderly people over 60 years $(6.28 \%)$ was about 1.5 times that of the middle-aged and young people. The prevalence of hypothyroidism in female over the age of 50 was $8 \%$ to $11 \%$, up to $21 \%$ when reaching 74 years or elder. Follow-up of 154 cases of female patients with hyperthyroidism found that, $1 / 3$ of subclinical hypothyroidism will turn into hypothyroidism 10 years later. Results of this study found that, the positive rate of female thyroid dysfunction was significantly different in different age groups $\left(\chi^{2}=15.457, \quad P=0.009\right)$, and the prevalence of subclinical hypothyroidism was the highest (4.38\%), while the prevalence of subclinical hypothyroidism in age group of larger than 40 was significantly higher than that of the age group of younger than 40 . The prevalence of male subclinical hypothyroidism increased along with the increase of age from 20 to 59 years old, reaching a peaking in the age of 50-59 $(4.36 \%)$. The prevalence of subclinical hypothyroidism in female was 5.41 times of that of male, accounting for $84.4 \%$ $(119 / 141)$. The results of the study were consistent with the prevalence of subclinical hypothyroidism in the general population of China of $0.91 \% \sim 6.05 \%$, increased with age, female predominated [19], and the study result of that the clinical and subclinical hypothyroidism risk may be increased with the iodine intake [20].

TPOAb and TGAb are the markers of secondary immune response after thyroid injury. It is an important index for the diagnosis of AITD and determination of etiology of primary hypothyroidism. The positive rate of serum TPOAb and $\mathrm{TgAb}$ in the general population were $10 \% \sim 15 \%$ and $3 \% \sim$ $11.5 \%$, respectively, reaching $11.8 \%$ and $11.9 \%$ in age of $30-40,20.2 \%$ and $22.4 \%$ in $60-70$, which were significantly higher in female than in male, low positive of two autoantibodies simultaneously [21]. Li Yushu et al [8] carried out a study on thyroid autoantibody in 3761 residents of 3 Chinese rural communities, and found that the positive rate of TPOAb and TGAb were $9.81 \%$ and $9.09 \%$, respectively, which in female $(11.71 \%, 11.21 \%)$ were significantly higher than those in male $(4.07 \%$ and $2.68 \%)$. A survey of 6112 residents showed that TPOAb and / or TgAb levels were elevated in $17.42 \%$ of female with normal serum thyroid hormone levels, approximately 2.91 times higher than male $(5.99 \%)$ [13]. The total positive rate of thyroid autoantibody in healthy subjects was $16.19 \%(521 / 3218)$, and the positive rate of $\mathrm{TPOAb}$ and $\mathrm{TgAb}$ was $14.57 \%$ and $12.74 \%$, respectively, which were consistent with results reported by $\mathrm{Li}$ Haiqiang et al [22], the positive rate of TPOAb and TgAb in adults were $15.0 \%$ and $13.0 \%$, respectively, and the positive rate of autoantibody was $17.96 \%$ in 1220 healthy subjects in Deyang, Sichuan Province [23], and higher than the results reported by Guan Haixia et al [7], that positive rate of TPOAb and $\mathrm{TgAb}$ were $11.6 \%$ and $9.3 \%$. The study also found that the positive rate of TPOAb in female was significantly higher than that in male $\left(21.53 \%\right.$ VS $\left.6.92 \%, \chi^{2}=137.634, P<0.001\right)$, with the peak appearing in the age equal to or larger than 70 $(28.10 \%)$, and the highest positive rate of TPOAb in male appeared in the age of 50-59 $(9.49 \%)$. The positive rate of $\mathrm{TGAb}$ in female was significantly higher than that in male (219.16\% VS 5.68\%, $\left.\chi^{2}=131.169, P<0.001\right)$, with the peak appearing in the age equal to or larger than 70 (22.22\%), and the highest positive rate of TGAb in male appeared in the age of 50-59 (7.32\%). The positive rate of TPOAb, TgAb in female was significantly higher than that in male, consistent with the above research results [8] [12] [13]. The simultaneous detection rate of TGAb and TPOAb in female was significantly higher than that in male (16.67\% VS 5.03\%, $\left.\chi^{2}=110.002, P<0.001\right)$, with the peak appearing in the age equal to or larger than $70(20.26 \%)$, and the highest simultaneous detection rate of TGAb and TPOAb in male appeared in the age of 50-59 $(5.15 \%)$. The positive rates of TPOAb, TGAb and simultaneous detection rate of TPOAb 
and $\mathrm{TgAb}$ in male and female and different age groups increased with the increase of age, of which the difference were statistically significant $(\mathrm{P}<0.001)$.

TPOAb and TGAb positive easily lead to thyroid dysfunction. Prummel et al [24] found that positive rate of $\mathrm{TPOAb}$ in population with normal thyroid function may reach $12 \%$ to $26 \%$, often accompanied by TGAb positive, of which positive rates were similar [25], and patients with high titers of TGAb were more likely to develop hypothyroidism [26]. In this study, the positive rates of TPOAb, TGAb and simultaneous detection rate of TPOAb and TgAb were $39.92 \%$ (99/248), 39.11\% (97/248) and 31.45\% (78/248) respectively in the patients with thyroid dysfunction, which is consistent with the results reported by Zhao Rencheng [27], subclinical hypothyroidism patients with autoantibody positive accounting for $1 / 3$ to $1 / 2$, indicating that thyroid autoantibody involved in the pathogenesis. The positive rate and OR of TPOAb, TGAb and simultaneous detection rate of TPOAb and $\mathrm{TgAb}$ of hypothyroidism were the highest, followed by hyperthyroidism, subclinical hypothyroidism and subclinical hyperthyroidism, which is consistent with the results in Beijing reported by Wen Xiaoheng et al [28], but the positive rate of autoantibody and OR of different thyroid diseases were different, for example, the positive rate and OR of TPOAb, TGAb and simultaneous detection rate of TPOAb and $\mathrm{TgAb}$ of subclinical hyperthyroidism and hyperthyroidism were higher than Beijing, while the TGAb of hyperthyroidism and simultaneous detection rate of TPOAb and $\mathrm{TgAb}$ and OR of hypothyroidism were lower than Beijing, which may be related to geographical area, number of study cases, sex ratio and diagnostic criteria.

TPOAb, TgAb-positive patients with subclinical hyperthyroidism is easy to evolve into hypothyroidism. A follow-up study of 4 to 8 years found that $20 \%$ to $50 \%$ of patients with subclinical hyperthyroidism would evolve into hypothyroidism. Patients who are larger than 65 years old, especially thyroid autoantibody-positive, the probability of subclinical hypothyroidism evolving into hypothyroidism within 4 years is up to $80 \%$ [29], indicating that thyroid autoantibody is a risk factor for thyroid dysfunction [30]. The simultaneous detection rate of TPOAb and TGAb is lower than that of any single antibody in population with thyroid dysfunction, but it is more likely to lead to the occurrence of AITD, indicating that the two antibodies play a synergistic role in the thyroid damage. Gao Jianhua et al [31] carried out follow-up on subjects with normal thyroid function but autoantibody-positive and found that the prevalence of hypothyroidism and hyperthyroidism after 1 year was 5.2\% and $3.7 \%$, respectively. The prevalence of hypothyroidism in TPOAb-positive patients $(5.5 \%)$ was significantly higher than that in TPOAb-negative patients $(31 \%)$ TPOAb $(0.5 \%)$, which is consistent with the results that subclinical hypothyroidism is more likely to occur in TPOAb and TGAb positive patients [32]. Whickham et al [33] had conducted a follow-up for 20 years and found that antibody-positive patient with normal thyroid function evolved into hypothyroidism at the rate of $2 \% \sim 4 \%$ every year. The peak of antibody appeared in the age period from 60 to 70 (70 excluded), 10 years earlier than the peak of incidence of hypothyroidism [28], confirmed that thyroid damage caused by autoantibody was a potential, chronic progressive process.

\section{Conclusion}

Cangzhou City, as a water-source-originated high level iodine area, with high detection rate of thyroid dysfunction and autoantibody-positive rate, especially the prevalence of subclinical hypothyroidism, not related to the sex and age. Usually subclinical hypothyroidism does not have obvious clinical manifestations [34], it is easy to be ignored, found mostly in thyroid function tests. TPOAb and TGAb were important indexes in the diagnosis of AITD. TPOAb in blood of AITD susceptible population will increase firstly, and then TSH increases, changes of FT4 and other thyroid hormone appear later. Therefore, it is important to incorporate the thyroid function and autoantibody testing as a routine physical examination of healthy people. Strengthen the screening of thyroid disease is conducive to early diagnosis and differential diagnosis, especially the dynamic monitoring and follow-up for autoantibody-positive patients with normal thyroid function will contribute to disease prevention and treatment.

\section{Acknowledgements}

This study was supported by a Natural Science Project of Cangzhou Medical College (No. 14Z001), a Science and Technology Program Fund Project of Hebei in China (No. 15277796D).

\section{References}

[1] Hou ZJ, Wang FL. Progress in immunological detection of thyroid hormones. Medical Recapitulate. 2016, vol. 22, pp. 332-335.

[2] Zhang Z, Xiao SB, Liu CY, et al. Analysis on clinical significance of A-TG and A-TPO detection. Labora tory Medicine, 2012, vol. 27, pp. 882-883.

[3] Ping LY, Du LS, Zhang ML. Thyroid disease diagnosis and treatment of $\mathrm{TpoAb}$, serum $\mathrm{TgAb}$ change and its value. Journal of Clinical and Experimental Medicine, 2016, vol. 15, pp. $1278-1281$.

[4] Ma JH, Mo QX, Yang YZ, et al. Different levels of thyroid autoimmune antibody in four kinds of thyroid diseases. Academic Journal Guang Zhou Med University, 2015, vol. 43, pp. 45-47.

[5] Dang L, Hu YT, Z ang YL. Application value of a variety of antibody detection in the diagnosis of thyroid diseases, China Med Herald, 2016, vol. 13, pp. 65-68.

[6] Shen HM, Zhang SB, Liu SJ, et al. Study on geographic distribution of national high water iodine areas ang the contours of water iodine in high iodine areas. Chin J Endemiol, 2007, vol. 26, pp. 658-661. 
[7] Guan HX, Teng WP, Cui BY, et al. An epidemiological survey of thyroid disorders in an area with high iodine content in water supply. Chin J Interm Med, 2001, vol. 40, pp. 597-601.

[8] Li YS, Shan ZY, Guan HX, et al. Determination and its clinical significance on the positive value of thyroid peroxidase antibody and thyroglobulin antibody. Chin J Lab Med, 2006, vol. 29, pp. 780-783.

[9] Dou YG, Wang YL, Li HB, et al. Iodine nutritional status and thyroid function of aduIts in urban and rural aress of Wuwei City Gansu Province. Chin J Endemiol, 2015, vol. 34, pp. 45-48.

[10] Teng WP, Xing XP, Dong NW, et al. epidemiology investigation of China's urban thyroid disease. The Chinese medical association proceedings of the ninth national conference on endocrine, dalian, Beijing: academic affairs department of the Chinese medical association. 2010.

[11] Yan YR, Liu Y, Huang H, et al. Iodine nutrition and thyroid diseases in Chengdu, China: an epidemiological study. QJM, 2014. pii: hcu216.

[12] ZHai ZC, Gao J. Study on the change of biological reference interval of thyroid function in middle aged and old people in Qishan. Labeled Immunoassys Clin Med, 2016, vol. 23, pp. 34-37.

[13] Yuan S, Jiang Lu, Zhu Li, et al. Investigation on the results of thyroid hormone and thyroid autoantibody determinations in 6112 healthy subjects in Shanghai. Laboratory Medicine, 2015, vol. 30 , pp. 219-213.

[14] Wen XH, Zhang B, Cai XF, et al. Analysis of the thyroid dysfunction screening in the checkup people. China Medical Herald, 2015, vol. 12, pp. 58-62.

[15] Wu XQ, Cao XY, Chen GW, et al. An epidemiological survey of thyroid disease among population aged over 18 in 3 districts of Xiamen City of Fujian Province. Chin J Endemiol, 2015, vol. 34, pp. 824-828.

[16] Cooper DS, Biondi B. Subclinical thyroid disease. Lancet, 2012, vol. 379 (9821), pp. 1142-1154.

[17] Eastman CJ. Screening for thyroid disease and iodine deficiency. Pathology, 2012, vol. 44, pp. 153-159.

[18] Canaris GJ, Manowitz NR, Mayor G, et al. The Colorado thyroid disease prevalence study. Arch Intern Med, 2000, vol. 160 , pp. 526-534.

[19] Liang P, Zhang Z, Dong Y. Effects of serum thyrotropin on serum lipid levels in elderly patients with subclinical hypothyroidism. Shandong Medical Journal, 2014, vol. 54, pp. 77-78.

[20] Hou CC, Liu ZH, Fu G. The research progress on the relationship between iodine and thyroid disease. Chin J Endemiol, 2015, vol. 34, pp. 824-828.

[21] Joseph GH, Norman WS, Dana F, et al. Serum TSH and thyroid antibodies in the Unites States populations (1988-1994): national health nutrition examination survey (NHANES III). J Clin Endocrinol Metab, 2002, vol. 87, pp. 489-499.

[22] Li HQ, Sang ZN, Tan L, et al. Thyroid function and serum lipids of adults living in areas of excessive iodine in Hebei Province. J Hygiene Res, 2012, vol. 41, pp. 536-539, 542.
[23] Ceng W. Thyroid function analysis of the results on Deyang area health check-up crowd West China Medical Journal, 2016, vol. 31, pp. 736-738.

[24] Prummel MF, Wiersinga WM. Thyroid peroxidase autoantibody in euthyroid subjects. Best Pract Res Clin Endocrinol Metab, 2005, vol. 19, pp. 1-15.

[25] Larsen PR. Williams textbook of endocrinology. Philadelphia: Sauders, 2003, pp. 360-365.

[26] Chou KM, Huang BY, Chen $\mathrm{CH}$, et al. Correlation and presentation of thyroid functional status with thyroid autoantibody in long-term follow-up of autoimmune thyroiditis: A study of 116 cases. J Formos Med Assoc, 2013, S0929-6646(13)00370-7.

[27] Zhao RC, Liu LX, Shen HM, et al. Comparison of detection rates of women's subclinical thyroid diseases between iodine-deficient regions with iodine supplying and water-borne iodine excess regions. Journal of Jilin University (Medicine Edition), 2014, vol. 40, pp. 668-674.

[28] Wen XH, Zhang B, Zhang Q, et al. Analysis of the relationship between thyroid antibodies and thyroid dis-function diseases in checkup people. China Medical Herald, 2015, vol. 12, pp. 69-72, 77.

[29] Wang F, Wen JH. Modern research progress of subclinical hypothyroidism. RESEARCH OF INTEGRATED TRADITIONAL CHINESE AND WESTERN MEDICINE, 2010, vol. 2, pp. 44-46.

[30] Luo ZW. Clinical research progress of thyroid autoantibody. YOUJIANG MEDICAL JOURNAL. 2004, vol. 32, pp. 278-280.

[31] Gao JH, Ma K, Yan XJ. Thyroid peroxidase antibodies and correlation study of subclinical hypothyroidism course of evolution. CHINA PRACTICAL MEDICAL. 2013, vol. 8, pp. 147-150.

[32] Caturegli P, De Remigis A, Rose NR. Hashimoto thyroiditis: clinical and diagnostic criteria. Autoimmun Res. 2014, vol. 13, pp. 391-397.

[33] DEMERS LM, SPENCER CA. Laboratory medicine practice guidelines: Laboratory support for the diagnosis and monitoring of thyroid disease. Clin Endocrinol (Oxf), 2003, vol. 58, pp. 138-140.

[34] Korevaar TI, Chaker L, Jaddoe VW, et al. Maternal and birth characteristics are determinants of offspring thyroid function. J Clin Endocrinol Metab, 2015, vol. 19, pp. 507-510.

\section{Biography}

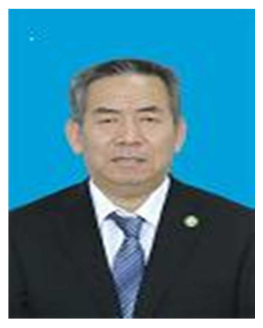

Hou Zhenjiang (1958-), male, with bachelor degree, Director of the Institute of Thyroid Disease, Professor, engaged in medical testing education and scientific research, the Hebei Province Higher Education Famous Teacher, medical and health assessment expert for A Guide to the Core Chinese periodical (2011 and 2014 Edition). 